\title{
Rhinoentomophthoramycosis: Madras ENT Research Foundation experience
}

\author{
Kiran Natarajan*, Sowmya Gajapathy, Swathi R., A. Soodimuthu, Mohan Kameswaran
}

Department of ENT, Madras ENT Research Foundation, Chennai, Tamil Nadu, India

Received: 10 August 2019

Accepted: 01 October 2019

\author{
*Correspondence: \\ Dr. Kiran Natarajan, \\ E-mail: kirannatarajan2001@yahoo.co.in
}

Copyright: (c) the author(s), publisher and licensee Medip Academy. This is an open-access article distributed under the terms of the Creative Commons Attribution Non-Commercial License, which permits unrestricted non-commercial use, distribution, and reproduction in any medium, provided the original work is properly cited.

\begin{abstract}
Rhinoentomophthoramycosis is a rare tropical fungal infection affecting the nasal cavities and the subcutaneous tissues. The most common causative organism is Conidiobolus coronatus followed by basidiobolus. In our institution, two patients with rhinoentomophthoramycosis were seen over a ten year period and were treated successfully with anti-fungal therapy. Rhinoentomophthoramycosis has to be considered in the differential diagnosis of a nasal granuloma or subcutaneous swellings in the face or neck.
\end{abstract}

Keywords: Rhinoentomophthoramycosis, Nasal lesion, Subcutaneous swellings

\section{INTRODUCTION}

Entomophthoramycosis is caused by Entomophthorales and includes conidiobolomycosis and basidiobolomycosis. In most patients, the infection is due to Conidiobolus coronatus. ${ }^{1}$ The infection in the nasal submucosa or in the subcutaneous tissues of the face results in nasal discharge, nasal obstruction, pain, nasal polyps or subcutaneous swellings of the lip or face. The overlying skin does not usually ulcerate. The diagnosis of entomophthoromycosis is mainly clinical or based on histopathological examination as culture may not be always be positive. Laboratory investigations of these patients are usually normal. Early identification and starting anti-fungal treatment results in good outcomes.

\section{CASE REPORT}

\section{Case 1}

A 60-year-old male presented with right nasal obstruction and a cheek lesion of 8 months duration. He had no significant personal or medical history. ENT examination revealed a lesion on the right side of the face (Figure 1) and an irregular, nodular lesion affecting the anterior part of the nasal septum, floor of the nose and the inferior turbinate on the right side. Biopsy of the nasal lesion was reported as entomophthoramycosis (Figure 2). On staining with lactophenol cotton blue, short, broad aseptate hyphae with large round conidia giving rise to secondary conidia were seen and the colony morphology was consistent with $C$. coronatus. He was started on liposomal amphotericin, to a total dose of 1.5 grams, and potassium iodide syrup (40 mg/kg/day) for three months after consultation with the infectious disease specialist. His lesions completely settled and he has had no recurrence in the last eight years.

\section{Case 2}

A 51-year-old male presented with right sided nasal obstruction, epistaxis, right facial pain and headache since six months. He had no significant personal or medical history. Diagnostic nasal endoscopy revealed a vascular lesion arising from the middle turbinate (Figure 3). The mucosa over the inferior turbinate, septum and the floor of the nose on the right side was boggy. Computerized tomography (CT) scans of the paranasal sinuses were done which showed a polypoidal mass attached to the right middle turbinate and nodular 
soft tissue masses in the subcutaneous plane over the right cheek with inflammation in the surrounding fat planes. Biopsy was reported as rhinoentomophthoramycosis. He was started on liposomal amphotericin to a total dose amounting to 1.5 grams, based on the mycologist's opinion. Subsequently nasal endoscopy showed a normal nasal cavity (Figure 4). He presented one year later with right facial and upper neck swelling since three weeks (Figure 5). He had no nasal symptoms. Diagnostic nasal endoscopy was unremarkable. He had a soft tissue mass of irregular margins in the subcutaneous plane adjacent to the right upper and lower jaw, right side of lower lip and in the submental and submandibular region. CT neck reported a lobulated soft tissue mass of well-defined irregular margins in the subcutaneous plane anterior and lateral to the right maxilla, extending inferiorly lateral to the mandible and into the submental and submandibular region with mild enhancement (Figure 6 and 7). Few right internal jugular and jugulodigastric lymphadenopathy was noted. The opinion of the mycologist was obtained. The patient was started on syrup posaconazole $300 \mathrm{mgs}$ twice daily for a period of three months as well as intravenous liposomal amphotericin to a total dose of 2 grams with periodic monitoring of renal and liver function. At the last followup, the patient was symptom free and has had no recurrence clinically in the last 3 months (Figure 8).

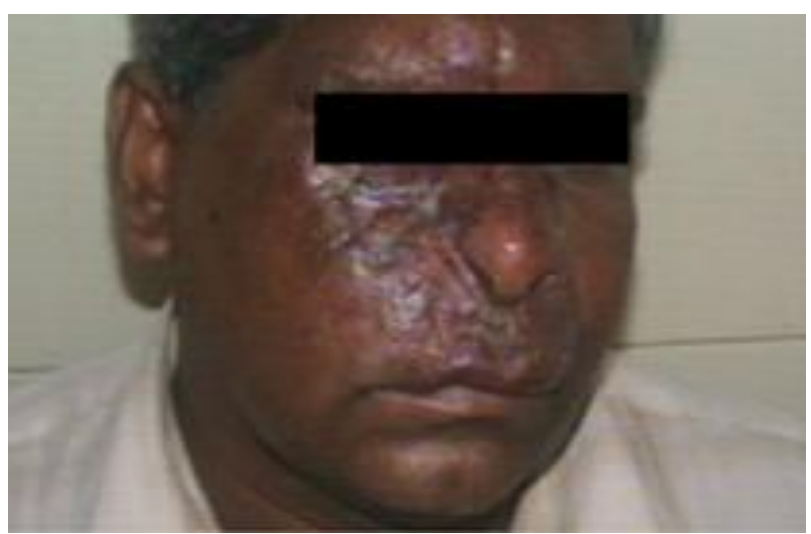

Figure 1: Right rhinofacial entomophthoramycosis.

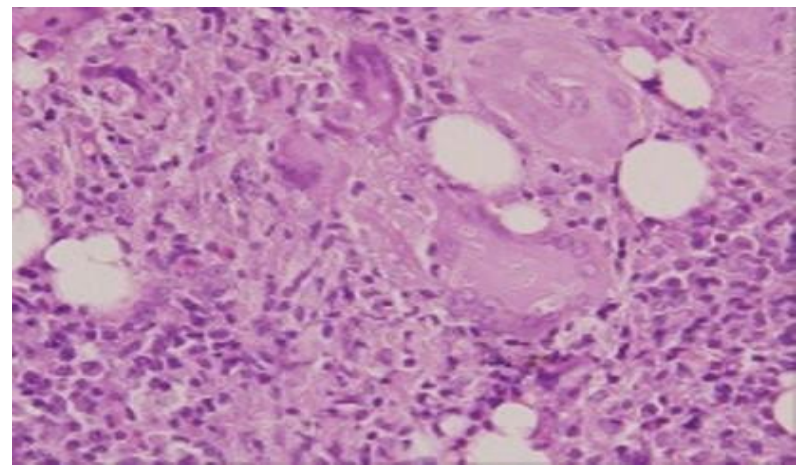

Figure 2: Granulomatous inflammation with multinucleated giant cells with well-defined tubular structures inside the giant cells.

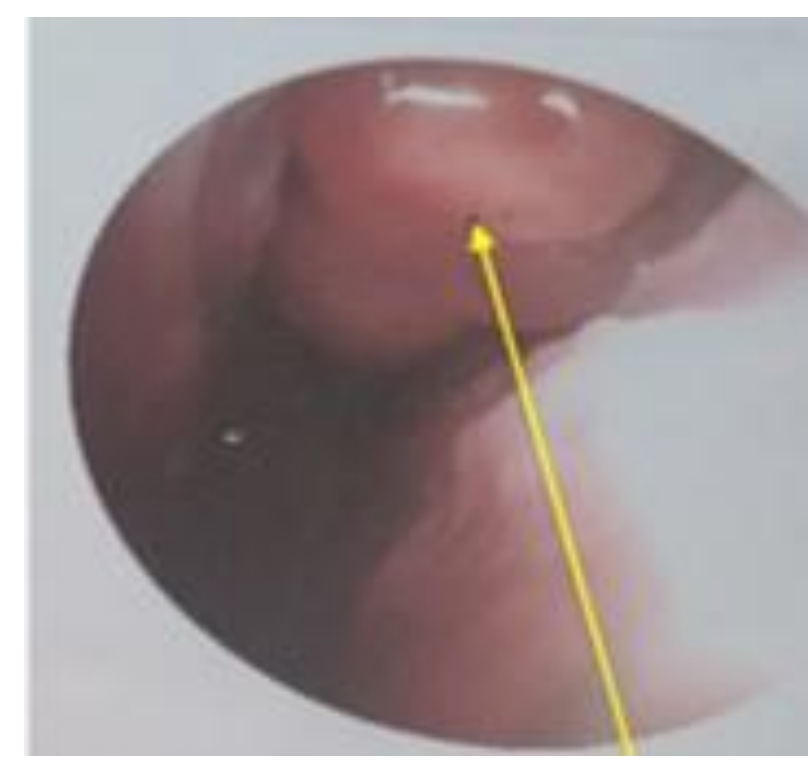

Figure 3: Polypoidal mass right nasal cavity.

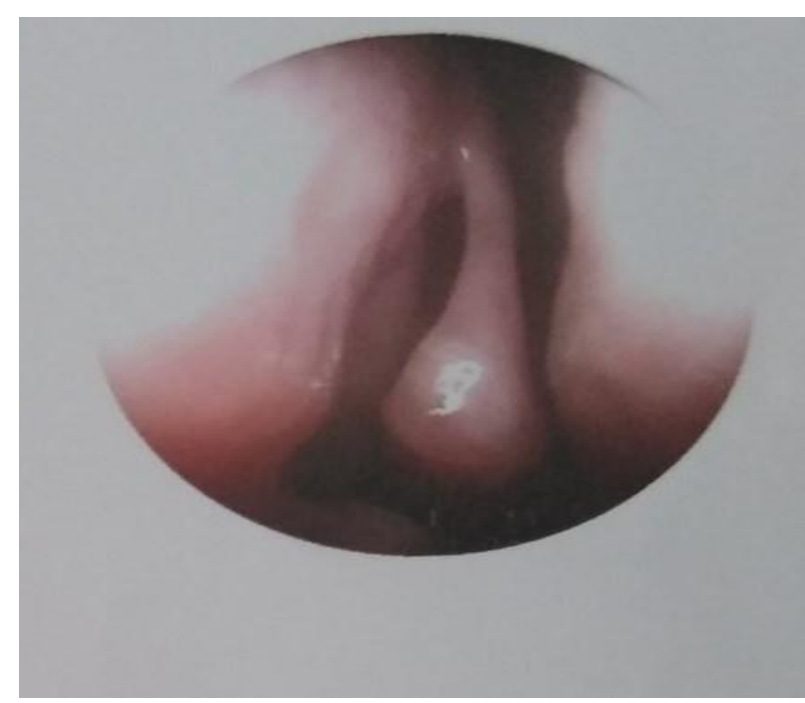

Figure 4: Resolution of entomophthoramycosis with anti-fungal treatment.

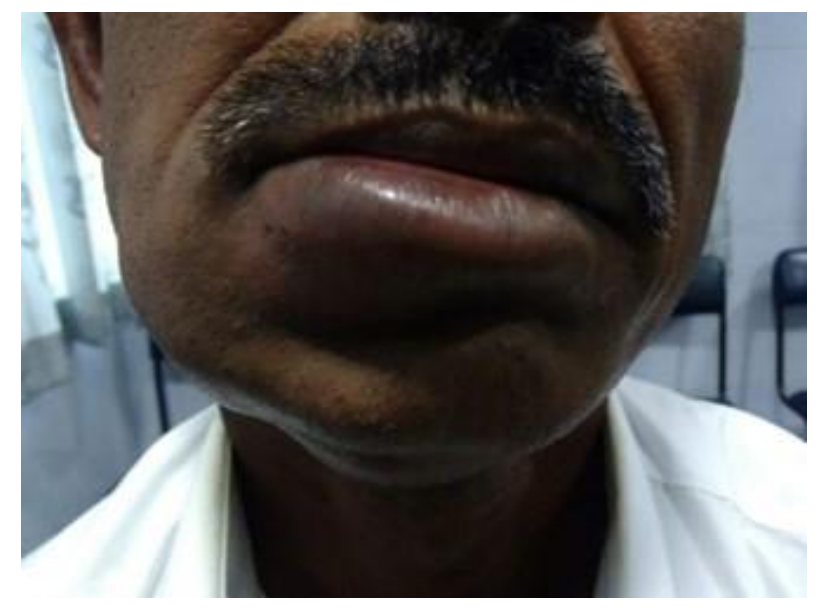

Figure 5: Recurrence of entomophthoramycosis one year later with subcutaneous swellings. 


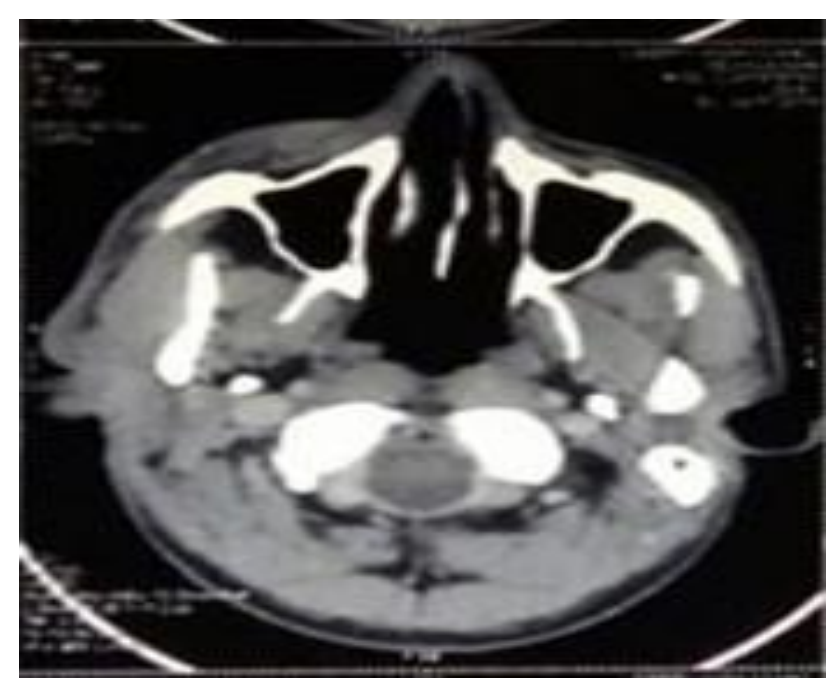

Figure 6: Recurrence of entomophthoramycosis with subcutaneous swellings lateral to the maxilla.

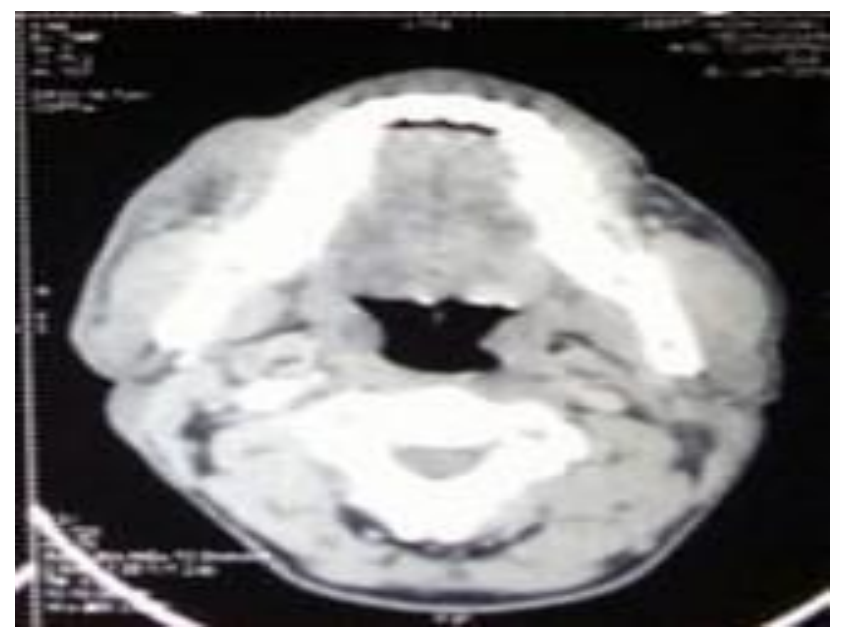

Figure 7: Recurrence of entomophthoramycosis with subcutaneous swellings lateral to the mandible.

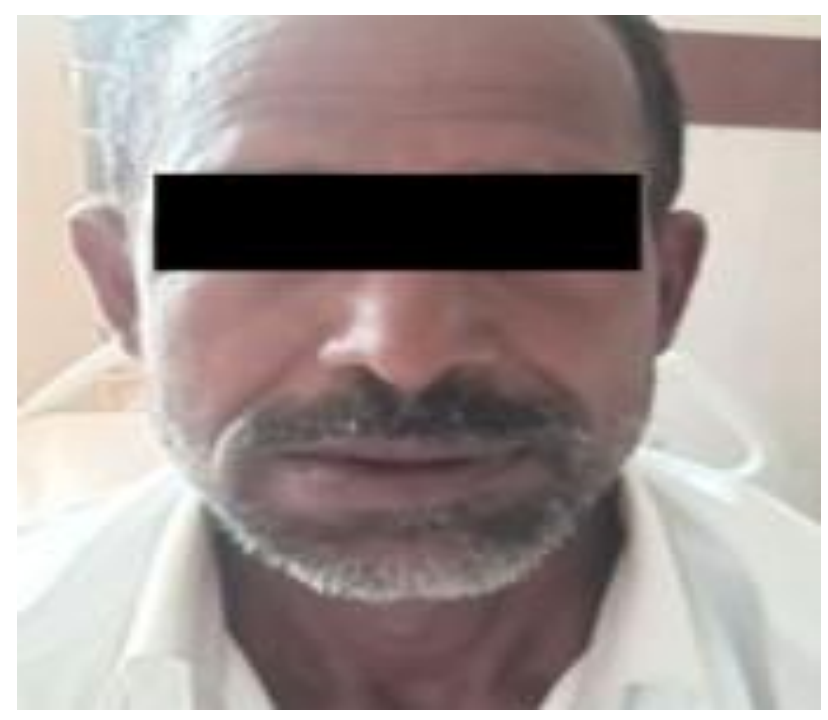

Figure 8: Resolution of subcutaneous entomophthoramycosis.

\section{DISCUSSION}

Entomophthoramycosis also known as entomophthoromycosis is a rare fungal infection in the tropical and subtropical regions of Africa, India and Latin America. $^{2}$ The word entomophthoramycosis translates in the Greek language to 'insect destroyer'. 3 Entomophthoramycosis most commonly causes chronic cutaneous and subcutaneous infections in immunocompetent individuals and presents in two forms: conidiobolomycosis (rhinofacial) and basidiobolomycosis (subcutaneous). This infection can cause disfigurement through scarring and lymphedema. In an immunocompromised host, they cause an infection with similar clinical, histopathological, and epidemiological features as that caused by mucorales fungi and may result in opportunistic pulmonary and disseminated infection. ${ }^{4}$ Patients present with features of recurrent sinusitis (fever, nasal obstruction, pain, mucosal hypertrophy of the sinuses, mid-face swelling or nasal mass). ${ }^{5}$ The first report of human infection by entomophthoramycosis was in 1965. Wide ecological distribution and rapid growth may be responsible for the development of human disease. These infections mostly occur in adult males. ${ }^{1}$ The fungus is introduced into the nasal mucosa by soiled hands or inhalation of conidia from environmental sources such as soil or decaying vegetable matter or insect bite. ${ }^{1}$

C. coronatus the commonest pathogen, is a saprophyte of decomposing vegetation. ${ }^{6}$ Lesions by $C$. coronatus begin as infiltration of the nasal mucosa and submucosa, extending to the adjacent tissues of the paranasal sinuses, nasal dorsum, upper lip, and face with a nodular infiltrative appearance. ${ }^{7}$ Usually the disease has a chronic benign clinical course. In advanced cases, the lesions can also affect the nasopharynx, oropharynx, palate, cervical region, and mediastinum. ${ }^{7}$ Conidiobolomycosis has been rarely reported to involve the nasolacrimal duct and orbit. ${ }^{8}$ Basidiobolus spp. more typically cause subcutaneous entomophthoramycosis of the limbs, back and thorax in immunocompetent patients. Cases of gastrointestinal infection caused by Basidiobolus spp. have been reported. ${ }^{3}$ No incubation period or spontaneous involution has been reported.

Diagnosis is made by clinical, histopathological and mycological examination. ${ }^{7}$ Direct microscopy of tissue in $\mathrm{KOH}$ shows broad nonseptate or irregularly branching, infrequently septate filamentous hyphae. ${ }^{6}$ Biopsy shows granulomatous infiltrate of histiocytes, eosinophils and multi-nucleated giant cells and polymorphonuclear cells. Broad thin walled infrequently septate hyphae surrounded by Splendore-Hoeppli phenomenon (periodic acid-Schiff stain-positive eosinophilic material surrounding fungal hyphae) and absence of vascular invasion and necrosis is pathognomonic of entomophthoramycosis. ${ }^{2}$ Grocott's methenamine silver (GMS) and Periodic acid-Schiff (PAS) stains have been reported to help identify the causative organism. Culture from biopsy tissue on Sabouraud glucose agar is useful to identify the fungus 
and large fluffy white, gray, or brownish colonies are seen. Intense fibrosis may make culture and identification of fungal elements on biopsy difficult. Fungal culture may be negative as the hyphae are fragile. ${ }^{8}$ Kaufman et al devised an immunodiffusion test to detect both Conidiobolus and Basidiobolus. ${ }^{9}$ Use of molecular methods with specific DNA probes and panfungal primers, as well as real time polymerase chain reaction (PCR) to detect and identify these organisms in tissue has been reported. ${ }^{3}$

Many antifungal drugs, such as potassium iodide (40 $\mathrm{mg} / \mathrm{kg} /$ day), amphotericin B (1 to $1.5 \mathrm{mg} / \mathrm{kg} /$ day $)$, imidazole derivatives (fluconazole $300 \mathrm{mg} / \mathrm{day}$ ), ketoconazole $(400 \mathrm{mg} /$ day $)$, and triazoles (itraconazole $300 \mathrm{mg} / \mathrm{day}$ ), or a combination of two of these drugs have been used for the treatment of rhinoentomophthoramycosis. ${ }^{2}$ Posaconazole can be used as salvage therapy in some patients who are refractory to, or intolerant to amphotericin B and its lipid derivatives. ${ }^{10}$ Posaconazole oral suspension, in a dosage of $800 \mathrm{mg}$ divided in two to four doses daily has been shown to be effective. Krishnan et al reported good success with potassium iodide at $40 \mathrm{mg} / \mathrm{kg} /$ day. ${ }^{11}$ In patients with either conidiobolomycosis or basidiobolomycosis, itraconazole or potassium iodide were reported to be reasonable first-line choices. ${ }^{8}$

Use of hyperbaric oxygen and itraconazole or miconazole after failure to produce clinical cure by amphotericin has been reported. Regular follow-ups are essential to monitor the response to treatment. No single agent has reliable consistent antifungal activity against entomophthoramycosis. In vitro susceptibility testing may be helpful to guide therapy. ${ }^{9}$ Blumentrath et al proposed a treatment strategy according to stages of disease progression. ${ }^{12}$ Rhinoentomophthoramycosis is classified into early, intermediate, late and atypical disease which provides information on the duration of antifungal treatment and the disease prognosis. ${ }^{12}$ Surgery is usually not curative but plays a role in the management of entomophthoramycosis, depending upon location, organism and extent of the infection. ${ }^{3}$

\section{CONCLUSION}

Entomophthoramycosis is a rare tropical mycotic infection causing sinonasal or cutaneous manifestations. Anti-fungal treatment has been reported to produce clinical and mycological cure. Follow-up of these patients should be emphasized and recurrence should be treated at the earliest. Otolaryngologists should be aware of this infection due to the increase in global travel in the past few decades.

\section{Funding: No funding sources}

Conflict of interest: None declared

Ethical approval: Not required

\section{REFERENCES}

1. Ribes JA, Vanover-Sams CL, Baker DJ. Zygomycetes in Human Disease. Clin Microbiol Rev. 2000;13(2):236-301.

2. Bittencourt AL, Marback R, Nossa LM. Mucocutaneous entomophthoramycosis acquired by conjunctival inoculation of the fungus. Am J Trop Med Hyg. 2006;75(5):936-8.

3. Shaikh N, Hussain KA, Petraitiene R, Schuetz AN, Walsh TJ. Entomophthoramycosis: a neglected tropical mycosis. Clin Microbiol Infect. 2016;22(8):688-94.

4. Coelho Filho JC, Pereira J, Rabello Junior A. Mediastinal and pulmonary entomophthoramycosis with superior vena cava syndrome: a case report. Rev Inst Med Trop Sao Paulo. 1989;31:430-3.

5. Mahajan VK, Chauhan PS, Mehta KS, Abhinav C, Sharma V, Thakur K. Subcutaneous phaeohyphomycosis of the face presenting as rhinoentomophthoramycosis. Int $\mathbf{J}$ Dermatol. 2013;52(9):1105-8.

6. Chattopadhyay S, Mukherjee T, Rit K, Chakraborty B, Ghosh P, Dasgupta A. Conidiobolomycosis: a case report. IOSR-JDMS. 2016;15(1):65-6.

7. Valle ACF, Wanke B, Lazéra MS, Monteiro PCF, Viegas ML. Entomophthoramycosis by Conidiobolus coronatus. Report of a case successfully treated with the combination of itraconazole and fluconazole. Rev Inst Med Trop Sao Paulo. 2001;43(4):233-6.

8. Arora P, Sardana K, Madan A, Khurana N. An old woman with a lump. Indian $\mathrm{J}$ Dermatol. 2016;61:697-9.

9. Prabhu RM, Patel R. Mucormycosis and entomophthoramycosis: a review of the clinical manifestations, diagnosis and treatment. Clin Microbiol Infect. 2004;10(1):31-47.

10. Greenberg RN, Mullane K, van Burik JA, Raad I, Abzug MJ, Anstead G, et al. Posaconazole as salvage therapy for zygomycosis. Antimicrob Agents Chemother. 2006;50:126-33.

11. Krishnan SG, Sentamilselvi G, Kamalam A, Das KA, Janaki C. Entomophthoromycosis in India: A 4-year study. Mycoses. 1998;41:55-8.

12. Deak L, Mudalagiriyappa S, Ballin A, Saxton D, Chakrabarti A. A Rhinofacial Conidiobolus coronatus Fungal Infection Presenting as an Intranasal Tumour. Sultan Qaboos Univ Med J. 2018;18(4): e549-52.

Cite this article as: Natarajan K, Gajapathy S, Swathi R, Soodimuthu A, Kameswaran M.

Rhinoentomophthoramycosis: Madras ENT Research Foundation experience. Int J Otorhinolaryngol Head Neck Surg 2019;5:1732-5. 